\title{
DIMENSION OF COMPACT METRIC SPACES
}

\author{
ALEXANDER N. DRANISHNIKOV*
}

ABSTRACT. We give a survey of old and new results in dimension theory of compact metric spaces. Most of the relatively new results presented in the survey are based on the cohomological dimension approach. We complement the survey by stating the basics of cohomological dimension theory and listing some of its applications beyond the dimension theory.

\section{Contents}

1. Introduction

2. Equivalent definitions

3. Classic and new results

3.1. Dimension of the union

3.2. Dimension of the product

3.3. Hurewicz mapping theorem 7

3.4. Dimension raising maps

3.5. Stable intersection in $\mathbb{R}^{n} \quad 9$

3.6. Universal spaces 10

4. Cohomological dimension theory 11

4.1. Connection with the dimension 12

4.2. Bockstein Theory 12

4.3. Arithmetic of dimension theory 13

5. Infinite dimensional compacta 16

5.1. Cohomological approach 17

5.2. Generalized cohomology and dim 17

6. Connections with other areas 19

6.1. Convergence in the Gromov-Hausdorff space 19

6.2. Surgery on topological manifolds 20

6.3. Hilbert-Smith conjecture 21

6.4. Boundaries of groups 22

Date: April 21, 2017.

2000 Mathematics Subject Classification. Primary 55M30; Secondary 53C23, $57 \mathrm{~N} 65$.

Key words and phrases. dimension, cohomological dimension.

${ }^{*}$ Supported by NSF, grant DMS-1304627. 
6.5. Large scale dimension $\quad 22$

References $\quad 23$

\section{INTRODUCTION}

In 1911 Brouwer [13] proved that the $m$-cube $I^{m}$ and the $n$-cube $I^{n}$ aro not homeomorphic for $m \neq n$. In the proof he used the property of the $n$-cube that it does not admit an $\epsilon$-map for sufficiently small $\epsilon>0$ to an $(n-1)$-dimensional polyhedron. This property leads to a concept of dimension of compact metric spaces which coincides with the modern definition. Formally this property was adopted as the definition of dimension by Alexandroff [1] in 1930.

We note that dimension of compact metric spaces admits many nonobviously equal definitions. The first formal definition was given by Brouwer [14] in 1913 though informally the ideas of such definition were proposed by Poincare [80]. Brouwer called his dimension invariant Dimensionsgrad and denoted it as $D g$. He proved that $D g\left(I^{n}\right)=n$. Later when other definitions of dimension appeared and were widely accepted Brouwer believed [15, 16, 17, 18], that for compact metric spaces they coincide with his $D g$. It turs out to be that he was right but the proof of it appeared almost a century later in a work of Fedorchuk, Levin and Shchepin [50].

Other famous contributers to the formulation of the concept of dimension are Lebesgue, Urysohn, Menger, Hurewicz and Čech.

One of the pillars of dimension theory is the celebrated Brouwer Fixed Point theorem. Without the Brouwer Fixed Point theorem used explicitly or implicitly mere existence of topological spaces of dimension $n>1$ cannot be established. We illustrate it on the following definition of dimension (Alexandroff [2]): $\operatorname{dim} X \geq n$ if and only if $X$ admits an essential map onto the $n$-cube. We note again that for compact metric spaces all known definitions of dimension are equivalent. We recall that a continuous map $f: X \rightarrow I^{n}$ is essential if there is no continuous deformation fixed on the preimage $f^{-1}\left(\partial I^{n}\right)$ of the map $f$ to a map to the boundary $g: X \rightarrow \partial I^{n}$. The Brouwer Fixed Point theorem is equivalent to the fact that there is no retraction of $I^{n}$ onto the boundary $\partial I^{n}$. Thus, it implies that the identity map $i d: I^{n} \rightarrow I^{n}$ is essential and, hence, $\operatorname{dim} I^{n} \geq n$.

In this survey we consider only compact metric spaces (called compacta) since many deep results in dimension theory are based on the cohomological dimension theory and the latter is developed to full extend for compacta only. Still the cohomological dimension theory works 
for $\sigma$-compact spaces but for general separable metric spaces it has different features and is not completely developed yet [64],[46],[47].

\section{Equivalent Definitions}

Throughout this paper we assume that maps are continuous and spaces are separable metrizable. Most of the time they will be compact or locally compact. We recall that a compactum means a compact metric space. By dimension of a space $\operatorname{dim} X$ we assume the covering dimension.

2.1. Definition. The covering dimension of a topological space $X$ does not exceed $n, \operatorname{dim} X \leq n$, if for every open cover $\mathcal{U}$ of $X$ there is an open refinement $\mathcal{V} \prec \mathcal{U}$ of multiplicity $\leq n+1$. We say that $\operatorname{dim} X=n$ if $\operatorname{dim} X \leq n$ and the condition $\operatorname{dim} X \leq n-1$ does not hold true.

We recall that the refinement $\mathcal{V} \prec \mathcal{U}$ means that for each $V \in \mathcal{V}$ there is $U \in \mathcal{U}$ with $V \subset U$. The multiplicity of a cover $\mathcal{V}$ is the maximal number of elements of $\mathcal{V}$ having a common point.

A family $\mathcal{U}$ of subsets of a metric space $X$ is called an $\epsilon$-family if the diameter of each set in the family does not exceed $\epsilon$. An $\epsilon$-family which is a cover of $X$ is called an $\epsilon$-cover of $X$.

2.2. Theorem. For a compact metric space $X$ the following are equivalent

- (1) $\operatorname{dim} X \leq n$;

- (2) Given $\epsilon>0$, there is an open $\epsilon$-cover $\mathcal{U}=\mathcal{U}^{0} \cup \cdots \cup \mathcal{U}^{n}$ of $X$ such that each family $\mathcal{U}^{i}$ consists of disjoint open sets;

- (3) For every $\epsilon>0, X$ admits an $\epsilon$-map to an $n$-dimensional simplicial complex;

- (4) For any closed subset $A \subset X$ and a continuous map $f$ : $A \rightarrow S^{n}$ there is a continuous extension $\bar{f}: X \rightarrow S^{n}$;

- (5) $X$ does not admit an essential map onto $I^{n+1}$;

- (6) $X$ is the union of $n+1$ subsets of dimension 0;

- (7) $X$ admits a light map to $I^{n}$;

- (8) $X$ is is the image of a 0-dimensional compactum $Y$ under a map $f: Y \rightarrow X$ with $\left|f^{-1}(x)\right| \leq n+1$ for all $x \in X$;

- (9) $X$ is the limit of an inverse system of n-dimensional polyhedra.

By definition, $\epsilon$-map $f: X \rightarrow Y$ is a map with $\operatorname{diam} f^{-1}(y) \leq \epsilon$ for all $y \in Y$. A map with 0-dimensional point preimages is called light.

We note that a version of Theorem 2.2 holds for fairly general spaces, in particular, for separable metric spaces [49]. 
Let $A, B \subset X$ be a closed disjoint subsets. A closed subset $S \subset X$ is called a separator if $A$ and $B$ lie in different components of $X \backslash S$. It is called a cut if they lie in different continuum components. The continuum components of $Z$ are defined by the equivalence relation: $z \sim z^{\prime}$ if there is a continuum $C$ containing $z$ and $z^{\prime}$.

We recall that the dimensions Ind, ind, and $D g$ are defined inductively as $\operatorname{Ind}(\emptyset)=\operatorname{ind}(\emptyset)=D g(\emptyset)=-1$ and $\operatorname{Ind}(X) \leq n$ if for any two closed disjoint subsets $A$ and $B$ in $X$ there a separator $S$ with $\operatorname{Ind}(S) \leq n-1, \operatorname{ind}(X) \leq n$ if for any closed subset $A \subset X$ and a disjoint point $b \in X$ there a separator $S$ with $\operatorname{ind}(S) \leq n-1$, and $D g(X) \leq n$ if for any two closed disjoint subsets $A$ and $B$ in $X$ there a cut $S$ with $D g(S) \leq n-1$. The following is a classic result combined with the Fedorchuk-Levin-Schepin theorem [50].

2.3. Theorem. For a compact metric space $X$,

$$
\operatorname{dim} X=\operatorname{Ind}(X)=\operatorname{ind}(X)=D g(X) .
$$

The following property of dimension characterizing dim was extracted by Ostrand [78] from Kolmogorov's approach to the 13th Hilbert problem.

2.4. Theorem. For a compact metric space $\operatorname{dim} X \leq n$ if and only if for any $k \in \mathbb{N}$ and $\epsilon>0$, there is a family $\mathcal{U}=\mathcal{U}^{0} \cup \cdots \cup \mathcal{U}^{n+k}$ of open subsets of $X$ such that each family $\mathcal{U}^{i}$ consists of disjoint sets of diameter $<\epsilon$ and each $n+1$ subfamilies $\mathcal{U}^{i_{0}} \cup \cdots \cup \mathcal{U}^{i_{n}}$ form a cover of $X$.

\section{Classic And NeW Results}

One of the fundamental questions about dimension is how it behaves with respect to operations on spaces such as taking the union, taking the product or applying a continuous map. In the case of compact metric spaces we have a satisfactory answer to this question.

\subsection{Dimension of the union.}

3.1. Theorem. Suppose that a compactum $X=A \cup B$ is presented as the union of two subsets and one of them is closed. Then

$$
\operatorname{dim} X=\max \{\operatorname{dim} A, \operatorname{dim} B\} .
$$

This theorem is a special case of the following theorem proven by Menger and independently by Urysohn in the 20th: 
3.2. Theorem (Countable Union Theorem). Suppose that a compactum $X=\cup X_{i}$ is presented as a countable union of closed subsets. Then

$$
\operatorname{dim} X=\sup _{i}\left\{\operatorname{dim} X_{i}\right\} .
$$

If the subsets are not closed we have the following estimate:

3.3. Theorem (Urysohn-Menger Formula). Suppose that a compactum $X=A \cup B$ for general subsets $A, B \subset X$, then

$$
\operatorname{dim} X \leq \operatorname{dim} A+\operatorname{dim} B+1 .
$$

This inequality could be sharp: For the interval $I=[0,1]$,

$$
\operatorname{dim} I=\operatorname{dim}(I \cap \mathbb{Q})+\operatorname{dim}(I \backslash \mathbb{Q})+1,
$$

since both sets $I \cap \mathbb{Q}$ and $I \backslash \mathbb{Q}$ are 0-dimensional.

We obtained the following improvement of the Urysohn-Menger inequality in the case of compacta satisfying the formula $\operatorname{dim}(X \times X)=$ $2 \operatorname{dim} X$. We call them here dimensionally regular (compacta of type I in [64]).

3.4. Theorem. [37] Suppose that $X=A \cup B$ is a dimensionally regular compactum, then

$$
\operatorname{dim} X \leq \operatorname{dim}(A \times B)+1
$$

3.2. Dimension of the product. The following inequality for compact metric spaces can be easily derived form Alexandroff's definition of dimension (3) of Theorem 2.2:

\subsection{Theorem.}

$$
\operatorname{dim}(X \times Y) \leq \operatorname{dim} X+\operatorname{dim} Y .
$$

In 1930 Pontryagin [82] discovered that the logarithmic law

$$
\operatorname{dim}(X \times Y)=\operatorname{dim} X+\operatorname{dim} Y
$$

does not hold for compacta. Moreover, in the 40s Boltyanskii [10] discovered that it does not work even for the product of a compactum with itself (also see [60]).

Compacta $X$ that satisfy the law $\operatorname{dim}(X \times Y)=\operatorname{dim} X+\operatorname{dim} Y$ for all compacta $Y$ are called dimensionally full-valued. We call compacta that do not satisfy the equality $\operatorname{dim}(X \times X)=2 \operatorname{dim} X$ Boltyanskii compavcta (compacta of type II in [64]). We note that every locally contractible compactum is dimensionally regular [22] and there are locally contractible compacta which are not dimensionally full-valued (see Theorem 3.12). In view of Theorem 3.8 all polyhedra are dimensionally full-valued. 
3.6. Theorem. [64] For all $n$

$$
\operatorname{dim} X^{n}=n \operatorname{dim} X
$$

if $X$ is dimensionally regular compactum and

$$
\operatorname{dim} X^{n}=n \operatorname{dim} X-n+1
$$

if $X$ is Boltyanskii compactum.

3.7. Theorem. [37] Suppose that $X=A \cup B$ is a Boltyanskii compactum, then

$$
\operatorname{dim} X \leq \operatorname{dim}(A \times B)+2 .
$$

For every Boltyanskii compactum $X$ with $\operatorname{dim} X \geq 5$ there is a decomposition $X=A \cup B$ with $\operatorname{dim} X=\operatorname{dim}(A \times B)+2$.

We note that this theorem does not hold for non-compact metric spaces [76].

The logarithmic law for dimension of the product holds for all not necessarily compact spaces when one of the factors is a polyhedron:

3.8. Theorem (Morita Theorem).

$$
\operatorname{dim}(X \times I)=\operatorname{dim} X+1 .
$$

In the case of compact $X$ the interval can be replaced by any 1 dimensional continuum [64]:

3.9. Theorem. For any compactum $X$ and a continuum $C$,

$$
\operatorname{dim}(X \times C) \geq \operatorname{dim} X+1 .
$$

3.10. Corollary. For connected non-singleton compacta

$$
\operatorname{dim}(X \times Y) \geq \max \{\operatorname{dim} X, \operatorname{dim} Y\}+1 .
$$

The inequality from Theorem 3.9 does not hold for noncompact $X$. There is a 2-dimensional subset $W \subset \mathbb{R}^{3}$ and a continuum $C$ with $\operatorname{dim}(W \times C)=2[41]$.

It turns out that there is no restriction on dimension of the product of two compacta except Theorem 3.5 and Corollary 3.10:

3.11. Theorem. [22] For any $m, n \in \mathbb{N}$ and every integer $r$ satisfying $\max \{m, n\}+1 \leq r \leq m+n$ there are dimensionally regular compacta $X$ and $Y$ with $\operatorname{dim} X=m, \operatorname{dim} Y=n$, and $\operatorname{dim}(X \times Y)=r$.

We note that dimension of the product can behave badly even for locally contractible compacta.

3.12. Theorem. [22] There are 4-dimensional locally contractible compacta $X$ and $Y$ with $\operatorname{dim}(X \times Y)=7$. 
3.3. Hurewicz mapping theorem. There is the following generalization of Theorem 3.5 [59].

3.13. Theorem (Hurewicz Mapping Theorem). For a continuous map between compacta $f: X \rightarrow Y$,

$$
\operatorname{dim} X \leq \operatorname{dim} Y+\sup _{y \in Y}\left\{\operatorname{dim} f^{-1}(y)\right\}
$$

Since dimension of the product could be much smaller than the sum of the dimension of the factors (see Theotrem 3.11), the following is an improvement of the Hurewicz Mapping Theorem:

3.14. Theorem. [37],[38] For a continuous map of a dimensionally regular compactum $f: X \rightarrow Y$,

$$
\operatorname{dim} X \leq \sup _{y \in Y}\left\{\operatorname{dim}\left(Y \times f^{-1}(y)\right)\right\} .
$$

For a continuous map of a Boltyanskii compactum $f: X \rightarrow Y$,

$$
\operatorname{dim} X \leq \sup _{y \in Y}\left\{\operatorname{dim}\left(Y \times f^{-1}(y)\right)\right\}+1 .
$$

For every Boltyanskii compactum with $\operatorname{dim} X \geq 6$ there a continuous map $f: X \rightarrow Y$ with

$$
\operatorname{dim} X=\sup _{y \in Y}\left\{\operatorname{dim}\left(Y \times f^{-1}(y)\right)\right\}+1 .
$$

3.4. Dimension raising maps. The first dimension raising continuous map $\pi: I \rightarrow I^{2}$ of the interval onto the square was constructed by Peano more than a century ago. Since then the problem of raising dimension of nice spaces by nice maps was of a great interest.

Since dimension is a local property of a space, locally trivial bundles cannot raise dimension. In this subsection we discuss the problem of raising dimension for the next best classes of maps like Hurewicz fibrations, projections onto the orbit space for compact group actions, and cell-like maps. We recall that the projection onto the orbit space of a compact group action is an open map. If the action is free, it is a Hurewicz fibration.

First example of a raising dimension projection onto the orbit space for a free action of a compact group was constructed by Kolmogorov in 1934 [61]. It was an action of 2-adic integers on a Menger curve with the 2-dimensional orbit space. Further examples were constructed by Raymond and Williams [85],[86],[93].

Thus, Hurewicz fibrations between compacta can raise dimension. It turns out that the projection onto the orbit space can raise dimension even to infinity. 
3.15. Theorem. [43] For every prime $p$ and each $n=2,3, \ldots, \infty$, there is an action of the group $G=\prod_{i=1}^{\infty}(Z / p Z)$ on a two-dimensional compact metric space $X$ with the $n$-dimensional orbit space.

3.16. Theorem. [65] For each $n=2,3, \ldots, \infty$ there is a free action of a compact group $G$ on a one-dimensional compact metric space $X$ with the $n$-dimensional orbit space.

We recall that a set in $F \subset \mathbb{R}^{n}$ is called cellular if it can be presented as the intersection of nested sequence of topological cells. A compactum $F$ is called cell-like if it can be embedded into some $\mathbb{R}^{n}$ as a cellular set. A map of a manifold $f: M \rightarrow X$ is called cellular (cell-like) if all preimages $f^{-1}(x), x \in X$, are cellular (cell-like). Note that a cell-like map is always surjective. It is known that the cell-like maps between manifolds are exactly the limits of homeomorphisms. The image of a cell-like map of an $n$-manifold is a homology $n$-manifold whose covering dimension is equal to $n$ or $\infty$. Thus, if a cell-like map raises dimension, it raises it to infinity. First dimension raising cell-like map of a manifold $f: M^{n} \rightarrow X$ was constructed in [21] for $n=7$. Then the following improvements were obtained.

3.17. Theorem. [48] There is a cell-like map of a 5-dimensional manifold $f: M^{5} \rightarrow X$ that raises the dimension, $\operatorname{dim} X=\infty$.

3.18. Theorem. [33], [34] For every torsion element $\alpha$ in homology Ktheory of a closed $n$-manifold $M, n \geq 6$, there is a cell-like map $f$ : $M \rightarrow X$ with $f_{*}(\alpha)=0$. In particular, $\operatorname{dim} X=\infty$.

It is well-know that the Hilbert-Smith conjecture is equivalent to the problem whether the group of $p$-adic integers $A_{p}$ can act (freely) on a manifold. By Yang's theorem the dimension of the orbit of such action on an $n$-manifold might be equal $n+2$ or infinity. There are constructions of free actions of $A_{p}$ on Menger spaces. Also there is an example due to Floyd of a free $p$-adic action on a 2-dimensional cell-like set $C$ with the 2-dimensional orbit space [94].

3.19. Theorem. If there is a free $A_{p}$-action on a closed manifold $M$ with the infinite dimensional orbit space, then there exists a cell-like Hurewicz fibration which raises dimension.

Proof. Suppose that $A_{p}$ acts freely on $M^{n}$ with $\operatorname{dim}\left(M^{n} / A_{p}\right)=\infty$. We consider Borel's construction for this $A_{p}$-action on $M$ and Floyd's $A_{p}$-action on $C$ to obtain a Hurewicz fibration $p: M^{n} \times_{A_{p}} C \rightarrow M^{n} / A_{p}$ with the cell-like fiber $C$. The other Hurewicz fibration associated with the Borel's construction $q: M^{n} \times{ }_{A_{p}} C \rightarrow C / A_{p}$ has the fiber $M^{n}$. 
The Hurewicz Mapping Theorem gives an estimate $\operatorname{dim}\left(M^{n} \times_{A_{p}} C\right) \leq$ $\operatorname{dim} C / A_{p}+\operatorname{dim} M^{n}=n+2$.

3.5. Stable intersection in $\mathbb{R}^{n}$. We say that two maps $f: X \rightarrow Z$ and $g: Y \rightarrow Z$ have unstable intersection if for any $\epsilon>0$ there are $\epsilon$-approximations $f^{\prime}: X \rightarrow Z$ and $g^{\prime}: Y \rightarrow Z$ of $f$ and $g$ respectively with disjoint images $f^{\prime}(X) \cap g^{\prime}(Y)=\emptyset$. We always assume that $X$ is a compactum.

3.20. Theorem. Every two maps of compacta $f: X \rightarrow \mathbb{R}^{n}$ and $g: Y \rightarrow$ $\mathbb{R}^{n}, n \neq 5$, have unstable intersection if and only if $\operatorname{dim}(X \times Y)<n$.

The 'only if' direction of this theorem was proven in [42]. The 'if' direction of the theorem was proven in codimension $\neq 2$ for all $n$ in [29], in codimension 2 for $n=4$ in [39], and in codimension 2 for $n>5$ in $[66]$.

When we speak about unstable intersection of subsets we assume the unstable intersection of the inclusion maps. The following Alexandroff's theorem can be derived from definition (3) of Theorem 2.2 by using the dual triangulation of $\mathbb{R}^{n}$.

3.21. Theorem. For every $k$-dimensional compactum $X \subset \mathbb{R}^{n}$ there is an $(n-k)$-dimensional simplicial complex realized in $\mathbb{R}^{n}$ that has a stable intersection with $X$.

There is a natural question known as the Chogoshvili conjecture whether the $(n-k)$-dimensional complex in Alexandroff's theorem can be replaced by an affine $(n-k)$-plane. For general compacta there is a counterexample:

3.22. Theorem. [27] There exists a 2-dimensional compactum $X \subset \mathbb{R}^{4}$ which has unstable intersection with every affine 2-plane $\alpha \subset \mathbb{R}^{4}$.

There is a hope that the Chogoshvilli conjecture holds true when $X$ is a manifold. It is known to be true for $k \leq 2$.

3.23. Theorem. [45] For every locally contractible 2-dimensional compactum $X \subset \mathbb{R}^{n}$ there is an affine $(n-2)$-dimensional plane $\alpha \subset \mathbb{R}^{n}$ that has a stable intersection with $X$.

We conclude this subsection by the following theorem of Nöbeling which was rediscovered by Mardešic̀.

3.24. Theorem. [75],[72] Suppose that $X \subset \mathbb{R}^{m}=\prod_{i \in M} \mathbb{R}$ is an $n$ dimensional compact subset of the euclidean $m$-space. Then there is a subset $N \subset M$ of cardinality $n$ such that the projection of $X$ onto $\mathbb{R}^{n}=\prod_{i \in N} \mathbb{R}$ has dimension $n$. 
3.6. Universal spaces. The Cantor set is obtained from the interval $I=[0,1]$ by the infinite procedure of removing open mid third intervals. It can be described as the set of all numbers $x \in I$ which can be presented in the ternary numeral system without using 1 s. The $n$ dimensional Menger space $\mu^{n}$ can be described similarly as the subset of all $\bar{x}=\left(x_{1}, x_{2}, \ldots x_{2 n+1}\right) \in I^{2 n+1}$ such that for any $k$ at most $n$ numbers $x_{1}^{k}, \ldots, x_{2 n+1}^{k}$ equal 1 in ternary presentations $x_{i}=0 . x_{i}^{1} x_{i}^{2} x_{i}^{3} \ldots, x_{i}^{k} \in$ $\{0,1,2\}$.

3.25. Theorem. The $n$-dimensional Menger space $\mu^{n}$ is universal for n-dimensional compacta.

We say that a space $Z$ has the disjoint $n$-disk property $\left(D D^{n} P\right)$ if every two maps $f, g: D^{n} \rightarrow Z$ of the $n$-ball have unstable intersection. Clearly, $\mathbb{R}^{2 n+1}$ has $D D^{n} P$. Perhaps it is less clear but it is true that $\mu^{n}$ has $D D^{n} P$. The following criterion was proved by Bestvina in his Ph.D. thesis.

3.26. Theorem (Characterization of Menger spaces). [7] An $(n-1)$ connected and locally $(n-1)$-connected $n$-dimensional compact that satisfies the disjoint $n$-disc property is homeomorphic to the Menger space $\mu^{n}$.

We recall that the $n$-dimensional Nöbeling space $\nu^{n}$ can be defined as the subset of points $\bar{x}=\left(x_{1}, x_{2}, \ldots, x_{2 n+1}\right) \in \mathbb{R}^{2 n+1}$ such that at most $n$ coordinates $x_{i}$ are rational. It is known that $\nu^{n}$ is universal for the class of all $n$-dimensional separable metric spaces. Moreover, $\nu^{n}$ can be naturally embedded in $\mu^{n}$.

The following criterion was proved by Nagorko in his Ph.D. thesis [77] (see also [70] for a different proof).

3.27. Theorem (Characterization of Nöbeling spaces). An $(n-1)$ connected and locally $(n-1)$-connected $n$-dimensional complete metric space that satisfies the discrete disjoint n-disc property is homeomorphic to the Nöbeling space $\nu^{n}$.

Theorem 3.25 implies the following:

3.28. Theorem (Nobelin-Pontryagin). Every $n$-dimensional compactum can be embedded in $\mathbb{R}^{2 n+1}$.

It is well-know that the dimension of the euclidean space cannot be improved there even for polyhedra. Nevertheless for Boltyanskii compacta there is an improvement:

3.29. Theorem. [88],[40] Every $n$-dimensional Boltyanskii compactum can be embedded in $\mathbb{R}^{2 n}$. 
There is a long standing open problem which is due to Borsuk: Can every contractible and locally contractible $n$-dimensional compactum be embedded in $\mathbb{R}^{2 n}$ ?

We recall that contractible and locally contractible 1-dimesional compacta are called dendrites.

3.30. Theorem. [11],[90] Every n-dimensional compactum can be embedded into the product of $n+1$ dendrites.

The above result was preceded by Nagata's theorem: Every n-dimensional compactum can be embedded into the product of $n+1$ Menger curves. We note that not every $n$-dimensional compactum can be embedded in the product of $n$ Menger curves $\mu^{1}$. Namely, Koyama, Krasinkiewicz and Spiez proved the following:

3.31. Theorem. [62] For a closed subset $X \subset\left(\mu^{1}\right)^{n}$ with $H^{n}(X) \neq 0$ the rank of $H^{1}(X)$ must be at least $n$.

Thus, $S^{n}$ cannot be embedded in $\left(\mu^{1}\right)^{n}$.

Theorem 3.25 was extended to mappings of compacta.

3.32. Theorem. [19] For every $n \in \mathbb{N}$ there is a Serre $(n-1)$-fibration $p_{n}: \mu^{n} \rightarrow Q$ of the $n$-dimensional Menger space $\mu^{n}$ onto the Hilbert cube with fibers $\mu^{n}$ which is universal for the class of maps of $n$ dimensional compacta.

Here the universality means that for every map $f: Z \rightarrow Z^{\prime}$ of $n$-dimensional compactum $Z$ there are embeddings $j: Z \rightarrow \mu^{n}$ and $j^{\prime}: Z^{\prime} \rightarrow Q$ such that $p_{n} \circ j=j^{\prime} \circ f$. A map $p: X \rightarrow Y$ is called a Serre $k$-fibration if it possesses the homotopy lifting property for $k$ dimensional polyhedra. We note that a Serre 0-fibration over a locally path-connected space is an open map and a Serre $(-1)$-fibration is just a surjective map.

\section{Cohomological Dimension theory}

Let $G$ be an abelian group. The cohomology of a space for us are always reduced Cech cohomology. We recall that they are presentable as $H^{n}(X ; G)=[X, K(G, n)]$ by means of an Eilenber-McLane space $K(G, n)$ where $[X, Y]$ denotes the homotopy classes of maps from $X$ to $Y$. We recall that $K(G, n)$ is an ANR space with trivial homotopy groups in all dimension $i \neq n$ and with $\pi_{n}(K(G, n))=G$. It is unique up to homotopy equivalence. It can be taken to be an abelian topological group. Then it gives an abelian group structure on $[X, K(G, n)]$. 
The cohomological dimension $\operatorname{dim}_{G} X$ of a space $X$ with respect to the coefficient group $G$ is defined as

$$
\operatorname{dim}_{G} X=\sup \left\{n \mid H^{n}(X, A ; G) \neq 0, A \subset_{C l} X\right\} .
$$

This definition can be restated in terms similar to Definition (4) in Theorem 2.2: $\operatorname{dim}_{G} X \leq n$ if for every closed $A \subset X$ and every continuous map $f: A \rightarrow K(G, n)$ there is a continuous extension $\bar{f}: X \rightarrow K(G, n)$.

4.1. Connection with the dimension. Clearly, $\operatorname{dim}_{G} X \leq \operatorname{dim}_{\mathbb{Z}} X \leq$ $\operatorname{dim} X$. Note that $\operatorname{dim}_{G} X=0$ for a non-degenerate group $G$ if and only if $\operatorname{dim} X=0$.

4.1. Theorem (Alexandroff Theorem). For finite dimensional compacta

$$
\operatorname{dim} X=\operatorname{dim}_{\mathbb{Z}} X .
$$

There are examples of infinite dimensional compacta which have finite cohomological dimension [21]. It is an easy observation that if $\operatorname{dim}_{\mathbb{Z}} X=1$ then $\operatorname{dim} X=1$. Dydak and Walsh constructed a counterexample to the equality $\operatorname{dim} X=\operatorname{dim}_{\mathbb{Z}} X$ with $\operatorname{dim}_{\mathbb{Z}}(X \times X)=3$ [48].

4.2. Theorem (Edwards Theorem). [92] For every compactum $Y$ of cohomological dimension $\operatorname{dim}_{\mathbb{Z}} Y=n$ there is a compactum $X$ with $\operatorname{dim} X=n$ and a cell-like map $p: X \rightarrow Y$.

A map $f: X \rightarrow Y$ is called $G$-acyclic if $H^{*}\left(f^{-1}(x) ; G\right)=0$ for all $x \in X$. It is natural to regard the empty set cyclic. Thus, a $G$-acyclic map is always onto. Note that cell-like maps are $G$-acyclic for all $G$. Edwards Theorem was extended to an arbitrary coefficient group $G$. We state here the most natural cases and refer to [69] for a general result.

4.3. Theorem. (1) [20] For every finite group $G$ and every compactum $Y$ with $\operatorname{dim}_{G} Y=n$ there is a compactum $X$ with $\operatorname{dim} X=n$ and $a$ $G$-acyclic map $p: X \rightarrow Y$.

(2) [68] For every subgroup $G \subset \mathbb{Q}$ and every compactum $Y$ with $\operatorname{dim}_{G} Y=n, n \geq 2$, there is a compactum $X$ with $\operatorname{dim} X=n$ and $a$ G-acyclic map $p: X \rightarrow Y$.

4.2. Bockstein Theory. Let $\mathcal{P}$ denote the set of all primes. The Bockstein basis is the collection of groups $\sigma=\left\{\mathbb{Q}, \mathbb{Z}_{p}, \mathbb{Z}_{p^{\infty}}, \mathbb{Z}_{(p)} \mid p \in\right.$ $\mathcal{P}\}$ where $\mathbb{Z}_{p}=\mathbb{Z} / p \mathbb{Z}$ is the $p$-cyclic group, $\mathbb{Z}_{p^{\infty}}=\operatorname{dirlim} \mathbb{Z}_{p^{k}}$ is the $p$-adic circle, and $\mathbb{Z}_{(p)}=\{m / n \mid n$ is not divisible by $p\} \subset \mathbb{Q}$ is the $p$-localization of integers. 
4.4. Theorem (Bockstein Theorem). For every abelian group $G$ there is a collection $\sigma(G) \subset \sigma$ such that for any compactum $X$,

$$
\operatorname{dim}_{G} X=\sup \left\{\operatorname{dim}_{H} X: H \in \sigma(G)\right\} .
$$

We refer to [64] and [31] for the definition of $\sigma(G)$. Since $\sigma(\mathbb{Z})=$ $\left\{\mathbb{Z}_{(p)} \mid p \in \mathcal{P}\right\}$, the Bockstein theorem implies that for finite dimensional compacta $X$

$$
\operatorname{dim} X=\max \left\{\operatorname{dim}_{\mathbb{Z}_{(p)}} X \mid p \in \mathcal{P}\right\} .
$$

We call a compactum $X$ p-regular if

$$
\operatorname{dim}_{\mathbb{Z}_{(p)}} X=\operatorname{dim}_{\mathbb{Z}_{p}} X=\operatorname{dim}_{\mathbb{Z}_{p} \infty} X=\operatorname{dim}_{\mathbb{Q}} X
$$

and call it $p$-singular otherwise.

The restrictions on the values of cohomological dimension of a given compactum with respect to Bockstein groups usually are stated in the form of Bockstein's inequalities [64]. Here we formulate them in a form of the equality and the alternative (see [31]).

4.5. Theorem. For every prime $p$ and every $p$-singular compactum $X$

$$
\begin{array}{r}
\operatorname{dim}_{\mathbb{Z}_{(p)}} X=\max \left\{\operatorname{dim}_{\mathbb{Q}} X, \operatorname{dim}_{\mathbb{Z}_{p} \infty} X+1\right\} \quad \text { and } \\
\text { either } \operatorname{dim}_{\mathbb{Z}_{p} \infty} X=\operatorname{dim}_{\mathbb{Z}_{p}} X \text { or } \operatorname{dim}_{\mathbb{Z}_{p} \infty} X=\operatorname{dim}_{\mathbb{Z}_{p}} X-1 .
\end{array}
$$

In the first case of the alternative we call $X p^{+}$-singular and in the second, $p^{-}$-singular. Thus, the values of $\operatorname{dim}_{F} X$ for Bockstein fields $F \in\left\{\mathbb{Z}_{p}, \mathbb{Q}\right\}$ together with the $p$-singularity types of $X$ for $p \in \mathcal{P}$ determine the value $\operatorname{dim}_{G} X$ for all groups.

4.3. Arithmetic of dimension theory. The cohomological dimension of a compactum can be coded by means of the following peculiar arithmetics $\mathbb{S}$ called Schepin's arithmetics [87]. We enlarge the natural numbers $\mathbb{N}$ to $\mathbb{S}$ by taking three copies of each $n \in \mathbb{N}, n>0$, denoted as $n^{-}, n, n^{+}$. Thus, it is the set of naturals with a possible \pm decorations. We consider the following order on $\mathbb{S}$

$$
1^{-}<1<1^{+}<2^{-}<2<2^{+}<\cdots<n^{-}<n<n^{+}<(n+1)^{-} \ldots
$$

and extend the commutative addition from $\mathbb{N}$ to $\mathbb{S}$ by the rule

$$
m^{ \pm}+n=(m+n)^{ \pm}, \quad m^{+}+n^{+}=(m+n)^{+}, \quad m^{-}+n^{-}=(m+n)^{-},
$$

and $m^{-}+n^{+}=(n+m)^{-}$. We note that in $\mathbb{S}$ there is a multiplication by natural numbers which preserves the order.

We define the norm \|\|$: \mathbb{S} \rightarrow \mathbb{N}$ by the rule $\left\|n^{-}\right\|=\|n\|=n$ and $\left\|n^{+}\right\|=n+1$. 
Let $\mathcal{P}_{0}=\mathcal{P} \cup\{0\}$ be the set of all primes together with 0 . For a compactum $X$ of $\operatorname{dim} X>0$ we define a function $D_{X}: \mathcal{P}_{0} \rightarrow \mathbb{S}$ as follows: $D_{X}(0)=\operatorname{dim}_{\mathbb{Q}} X, D_{X}(p)=\operatorname{dim}_{\mathbb{Z}_{p}} X$ decorated by - if $X$ is $p^{-}$-singular, decorated by + if $X$ is $p^{+}$-singular, and undecorated if $X$ is $p$-regular. Since the equality $\operatorname{dim}_{G} X=0$ implies that $X$ is 0-dimensional, it follows that $D_{X}(p) \neq 1^{-}$for all $p$.

Note that $\operatorname{dim}_{\mathbb{Z}_{(p)}} X=\max \left\{\left\|D_{X}(p)\right\|, D_{X}(0)\right\}$. In view of Alexandroff theorem for finite dimensional $X$ we obtain $\operatorname{dim} X=\sup _{p \in \mathcal{P}}\left\|D_{X}(p)\right\|$.

The following theorem was proven by Bockstein in a different language.

4.6. Theorem. For compacta $X$ and $Y$,

(1) $D_{X \cup Y}=\max \left\{D_{X}, D_{Y}\right\}$;

(2) $D_{X \times Y}=D_{X}+D_{Y}$.

It follows from the definition that the function $D_{X}$ satisfies the conditions

$$
D_{X}\left(\mathcal{P}_{0}\right) \cap \mathbb{N}=\left\{D_{X}(0)\right\} .
$$

4.7. Definition. We call a function $D: \mathcal{P}_{0} \rightarrow \mathbb{S} \backslash\left\{1^{-}\right\}$a dimension type if $D\left(\mathcal{P}_{0}\right) \cap \mathbb{N}=\{D(0)\}$.

4.8. Theorem (Realization Theorem). [26] For every dimension type function $D: \mathcal{P}_{0} \rightarrow \mathbb{S}$ there is a compactum $X$ such that $D=D_{X}$.

We note that the Realization Theorem together with the Bockstein theorem (Theorem 4.6) are the main ingredients of the proof of Theorem 3.11 .

4.9. Theorem. Suppose that $\operatorname{dim} X<\infty$.

(1) A compactum $X$ is dimensionally full-valued if and only if $D_{X}(\mathcal{P}) \subset$ $\mathbb{N}$. Thus, $D_{X}(p)=D_{X}(0)=\operatorname{dim} X$ for all $p$.

(2) A compactum $X$ is Boltyanskii if and only if the maximal value of $D_{X}$ has decoration + .

Proof. (1) If $D_{X}(p) \in \mathbb{N}$ for all $p$, then

$$
\operatorname{dim}(X \times Y)=\max \left\{\left\|D_{X \times Y}(p)\right\|\right\}=\max \left\{\left\|D_{X}(0)+D_{Y}(p)\right\|\right\}=
$$

$\max \left\{\operatorname{dim} X+\left\|D_{Y}(p)\right\|\right\}=\operatorname{dim} X+\max \left\{\left\|D_{Y}(p)\right\|\right\}=\operatorname{dim} X+\operatorname{dim} Y$. Suppose that $D_{X}(p)=n^{ \pm}$for some $p$. We may assume that $\operatorname{dim} X>1$. Consider the dimension type $D$ with $D(q)=1$ for $q \neq p$ and $D(p)=m^{+}$ where $m=\operatorname{dim} X-1$. By Realization Theorem there is a compactum $Y$ such that $D=D_{Y}$.

If $D_{X}(p)=n^{+}$, then $\operatorname{dim}(X \times Y)=\max _{r \in \mathcal{P}}\left\{\left\|D_{X}(r)+D_{Y}(r)\right\|\right\}=\max _{q \neq p}\left\{\left\|D_{X}(q)\right\|+1,\left\|n^{+}+m^{+}\right\|\right\} \leq$ 
$\max \{\operatorname{dim} X+1, n+m+1\}=n+m+1<\left\|D_{X}(p)\right\|+\operatorname{dim} Y \leq \operatorname{dim} X+\operatorname{dim} Y$.

Hence $X$ is not dimensionally full-valued.

If $D_{X}(p)=n^{-}$, then

$\operatorname{dim}(X \times Y)=\max _{r \in \mathcal{P}}\left\{\left\|D_{X}(r)+D_{Y}(r)\right\|\right\}=\max _{q \neq p}\left\{\left\|D_{X}(q)\right\|+1,\left\|n^{-}+m^{+}\right\|\right\} \leq$

$\max \{\operatorname{dim} X+1, n+m\}=n+m<\left\|D_{X}(p)\right\|+\operatorname{dim} Y \leq \operatorname{dim} X+\operatorname{dim} Y$.

Again, we obtain that $X$ is not dimensionally full-valued.

(2) If the maximal value of $D_{X}$ is $n$ or $n^{-}$, then $\operatorname{dim} X=n$ and $\operatorname{dim}(X \times X)=\max \left\{\left\|2 D_{X}(p)\right\|\right\}=2 n$. Thus, $X$ is not a Boltyanskii compactum. If the maximal value of $D_{X}$ is $n^{+}$, then $\operatorname{dim} X=n+1$ and $\operatorname{dim}(X \times X)=\max \left\{\left\|2 D_{X}(p)\right\|\right\}=2 n+1$. So $X$ is a Boltyanskii compactum.

There is a natural involution $*$ on $\mathbb{S}$ which fixes $\mathbb{N}$ and exchange the decorations: $\left(n^{+}\right)^{*}=n^{-}$and $\left(n^{-}\right)^{*}=n^{+}$. It defines the involution on dimension types. The involution on $\mathbb{S}$ does not commute with the addition, nevertheless it plays an important role in the cohomological dimension theory. In particular, it plays a crucial role in the stable intersection theorem (Theorem 5.9). Also it takes a part in the following Splitting Theorem [26],[37]:

4.10. Theorem. Let $D_{1}$ and $D_{2}$ be dimension types and let $X$ be a compactum that satisfies the inequality $D_{X} \leq\left(D_{1}^{*}+D_{2}^{*}\right)^{*}+1$. Then there is a decomposition $X=A \cup B$ with $D_{A} \leq D_{1}$ and $D_{B} \leq D_{2}$.

We note that the definition of $D_{X}$ can be applied when $X$ is not compact.

4.11. Corollary ( $p$-Splitting of the $n$-space). For every $n$ and every prime $p$ there is a decomposition $I^{n}=A \cup B$ with $\operatorname{dim}_{\mathbb{Z}_{p}} A=1$ and $\operatorname{dim}_{\mathbb{Z}\left[\frac{1}{p}\right]} B=1$.

The converse implication is also true:

4.12. Theorem. Suppose that a compactum $X$ is presented as the union $X=A \cup B$ with $D_{A} \leq D_{1}$ and $D_{B} \leq D_{2}$. Then $D_{X} \leq\left(D_{1}^{*}+D_{2}^{*}\right)^{*}+1$.

This is a special case of Dydak's Union Theorem [47] (see [37] for details). It lies in the heart of the improvement of the Urysohn-Menger formula presented in Theorem 3.4.

We notice that the Alexandroff theorem, the Bockstein theorem, and Theorem 4.5 imply the following.

4.13. Corollary. For every finite dimensional compactum $X$ there is a field $F$ such that $\operatorname{dim} X \leq \operatorname{dim}_{F} X+1$. 
For dimensionally regular compatca $X$ with $\operatorname{dim} X<\infty$ there is a field $F$ such that $\operatorname{dim} X=\operatorname{dim}_{F} X$. Note that for coefficients in a field there is the formula

$$
\operatorname{dim}_{F}(X \times Y)=\operatorname{dim}_{F} X+\operatorname{dim}_{F} Y .
$$

\section{INFINITE DIMENSIONAL COMPACTA}

A map $f: X \rightarrow Q=\prod_{i=1}^{\infty} I$ to the Hilbert cube is called essential if the composition $p_{n} \circ f: X \rightarrow I^{n}$ is essential for every projection $p_{n}: Q \rightarrow I^{n}$ onto the factor. A compact space $X$ is called strongly infinite dimensional if it admits an essential map onto the Hilbert cube. We note that this definition of strongly infinite dimensional spaces is good only for compact spaces. For general spaces one should use essential families of subsets [49]. A compactum $X$ is called hereditary (strongly) infinite dimensional if every closed subset of it is either (strongly) infinite dimensional or 0-dimensional.

5.1. Theorem. [57] Every strongly infinite dimensional compactum contains a hereditary strongly infinite dimensional compactum.

The following result of Levin solves a seemingly unreachable open problem from [51]. It is heavily based on the cohomological dimension theory.

5.2. Theorem ([67]). The product of three hereditary infinite dimensional compacta is never hereditary infinite dimensional.

An infinite dimensional space which is not strongly infinite dimensional is called weakly infinite dimensional (WID). It is convenient to include all finite dimensional spaces to the class of weakly infinite dimensional. There are the following inclusions of classes of compacta

$$
\text { countably dimensional } \subset \text { property } C \subset W I D \text {. }
$$

The first inclusion is proper in view of Pol's example [81]. The properness of the second inclusion is still an open question. We recall the definitions.

5.3. Definition. A compactum $X$ has property $C$ if for any sequence $\epsilon_{i}>0$ there is $n \in N$ and an open cover $\mathcal{U}$ of $X$ which can be presented as the union of $n$ disjoint $\epsilon_{i}$-families $\mathcal{U}=\mathcal{U}^{1} \cup \cdots \cup \mathcal{U}^{n}, i=1, \ldots, n$.

A compactum is called countably dimensional if it can be presented as the countable union of 0-dimensional (not necessarily closed) subsets.

The following theorem was proven by R. Ancel [4].

5.4. Theorem. For compacta with property $C$ there is the equality $\operatorname{dim} X=\operatorname{dim}_{\mathbb{Z}} X$. 
Since there exist infinite dimensional compacta with finite cohomological dimension, in view of Ancel's theorem the following is not surprizing.

5.5. Theorem ([23]). There are strongly infinite dimensional compacta with finite cohomological dimension $\operatorname{dim}_{\mathbb{Z}}$.

5.1. Cohomological approach. We call a relative cohomology class $\alpha \in H^{k}(X, A)$ pure relative if $j^{*}(\alpha)=0$ for the inclusion of pairs homomorphism $j^{*}: H^{k}(X, A) \rightarrow H^{k}(X)$. This means that $\alpha=\delta(\beta)$ for $\beta \in \tilde{H}^{k-1}(A)$. We call a space $X$ cohomologically strongly infinite dimensional if there is a sequence of pure relative classes $\alpha_{i} \in$ $H^{k_{i}}\left(X, A_{i}\right), k_{i}>0, i=1,2, \ldots, A_{i} \subset_{C l} X$ such that the cup-product $\alpha_{1} \cup \alpha_{n} \cup \cdots \cup \alpha_{n} \neq 0$ for all $n$. One can take any coefficient ring $R$ to define cohomologically strongly infinite dimensional compacta with respect to $R$.

5.6. Theorem. [3] Cohomologically strongly infinite dimensional compacta over any coefficient ring $R$ are strongly infinite dimensional.

Compacta which are not cohomologically strongly infinite dimensional are called compacta with weak relative cohomology over $R$. Thus, the class of compacta with weak relative cohomology over $R$ contains weakly infinite dimensional compacta as well as all compacta with finite cohomological dimension over $R$.

5.7. Theorem. [3] For any compactum $X$ with weak relative cohomology over a ring $R$ and any embedding into the Hilbert cube $X \subset Q$ the reduced $R$-homology groups of the complement are trivial,

$$
\tilde{H}_{*}(Q \backslash X ; R)=0 .
$$

This fact was proven for finite dimensional $X$ in [63] and for weakly infinite dimensional $X$ in [54].

5.2. Generalized cohomology and dim. We refer to [91] for the treatment of generalized cohomology theories and spectra. Here we briefly recall that spectrum $E$ is a sequence of CW-complexes $\left\{E_{n}, s_{n}\right.$ | $n \in \mathbb{Z}\}$ and cellular embeddings $s_{n}: S E_{n} \rightarrow E_{n+1}$ where $S$ is the redused suspension. The reduced cohomology theory $\tilde{h}^{*}$ is defined by a spectrum $E$ as follows: $\tilde{h}^{*}(X)=\lim _{\rightarrow}\left[S^{k} X, E_{n+k}\right]$. The nonreduced cohomology can be restored from reduced by the standard way $h^{*}(X, A)=\tilde{h}^{*}\left(X^{+} / A^{+}\right)$. For a spectrum $E$ we use notation $H^{*}(X ; E)$ for the corresponding cohomology.

For an abelian group $G$ we denote the Eilenberg-Mclane spectrum $\{K(G, n)\}$ by $G$. By $\mathbb{S}$ we denote the spherical spectrum $\left\{S^{n}, S\right\}$ and 
by $\pi_{s}^{*}$ the corresponding cohomology theory, called the stable cohomotopy groups. A spectrum with the property $E_{n}=\{p t\}$ for $n<0$ as well as corresponding (co)homology theory is called connective. It is known that every generalized cohomology theory has its connective counterpart. Every cohomology theory can be defined by an $\Omega$ spectrum where each $E_{n}$ is homotopy equivalent to the loop spaces $\Omega E_{n+1}$. In the case of an $\Omega$-spectrum $E$ we have for the reduced cohomology $\tilde{H}^{n}(X ; E)=\left[X, E_{n}\right]$ where $\left[X, E_{n}\right]$ denotes the set of pointed homotopy classes of maps of $X$ to $E_{n}$.

For a connective spectrum $E$ one can define the cohomological dimension

$$
\operatorname{dim}_{E} X=\sup \left\{n \mid H^{n}(X, A ; E) \neq 0, A \subset_{C l} X\right\} .
$$

5.8. Theorem. [23] For any connective spectrum $E$ and any compact metric space $X$,

$$
\operatorname{dim}_{\mathbb{Z}} X \leq \operatorname{dim}_{E} X \leq \operatorname{dim} X .
$$

We note that for any connective spectrum $E$ there is an inequality $\operatorname{dim}_{E} X \leq \operatorname{dim}_{\mathbb{S}} X$. For finite dimensional $X$ we have $\operatorname{dim}_{E} X=\operatorname{dim} X$ for all connective spectra.

5.9. Theorem. [24] For strongly infinite dimensional compacta $\operatorname{dim}_{\mathbb{S}} X=$ $\infty$.

There is a natural conjecture that $\operatorname{dim} X=\operatorname{dim}_{\mathbb{S}} X$ for all compacta. The obstacle there can be reduced to the problem about coincidence of the class of compacta with property $C$ with the class of weakly infinite dimensional compacta: $W I D \stackrel{?}{=}$ property $C$.

5.10. Corollary. If $W I D=$ property $C$, then the covering dimension coincides with the stable cohomotopy dimension, $\operatorname{dim} X=\operatorname{dim}_{\mathbb{S}} X$.

Proof. By Ancel's theorem for compacta with property C we obtain the inequality $\operatorname{dim} X=\operatorname{dim}_{\mathbb{Z}} X \leq \operatorname{dim}_{\mathbb{S}} X$. By Theorem 5.8 we have the equality there. Then Theorem 5.9 completes the proof.

There are special spectra called ring spectra for which one can define the cup product. Examples of ring theories include the ordinary cohomology with coefficients in a ring, $\pi_{s}^{*}$, and K-theories: periodic $K^{*}$, $K O^{*}$, and connective $k u^{*}, k o^{*}$ (possibly with coefficients). For ring spectrum $E$ one can define the cup-length dimension

$\cup-\operatorname{dim}_{E} X=\sup \left\{n \mid \alpha_{1} \cup \cdots \cup \alpha_{n} \neq 0, \alpha_{i} \in \tilde{H}^{k_{i}}\left(X, A_{i} ; E\right), A_{i} \subset_{C l} X\right\}$.

5.11. Theorem. For any ring spectrum $E$ and all compacta $X$,

$$
\cup-\operatorname{dim}_{E} X \leq \operatorname{dim} X \text {. }
$$


For connective ring spectra

$$
\cup-\operatorname{dim}_{E} X \leq \operatorname{dim}_{E} X
$$

Proof. Let $n=\operatorname{dim} X$. Using approximation of $X$ by an inverse sequence of $n$-dimensional polyhedra (see Theorem $2.2(9)$ ) we can reduce the statement to the case when $X$ is a polyhedron.

Let

$$
w=\alpha_{1} \smile \cdots \smile \alpha_{k} \neq 0
$$

where $\alpha_{i} \in \tilde{H}^{n_{i}}\left(X / A_{i} ; E\right), i=1, \ldots, k$, and $k>n$ for closed sets $A_{i} \subset X$. We may assume that $E$ is an $\Omega$-spectrum. Let $f_{i}: X \rightarrow E_{n_{i}}$ generate $\alpha_{i}$. Then $w$ is generated by the map

$$
X \stackrel{\Delta}{\longrightarrow} X \wedge \cdots \wedge X \stackrel{f_{1} \wedge \cdots \wedge f_{k}}{\longrightarrow} E_{n_{1}} \wedge \cdots \wedge E_{n_{k}} \longrightarrow E_{n_{1}+\cdots+n_{k}} .
$$

We may assume that the spaces $Z_{i}=f_{i}(X)$ are connected ANRs. Then the map $f_{1} \wedge \cdots \wedge f_{k}$ factors through a $n$-connected ANR-space $Z_{1} \wedge \cdots \wedge Z_{k}$ and hence is null-homotopic.

The second statement follows from the definition of $\operatorname{dim}_{E}$.

5.12. Theorem. For finite dimensional $X$,

$$
\cup-\operatorname{dim}_{\mathbb{Z}} X=\operatorname{dim} X \text {. }
$$

Proof. We apply (5) of Theorem 2.2 to get the result.

Using a ring spectrum $E$ one can define cohomologically strongly infinite dimensional comapcta with coefficients in $E$ and prove an analog of Theorem 5.6.

\section{Connections With other areas}

In this section we give a quick sketch of some applications of the dimension theory to topology of manifolds and to geometric group theory. We leave out the picture its applications to functional analysis such as in [89] or [25], logic (see [97]), dynamical systems ([53],[71]).

6.1. Convergence in the Gromov-Hausdorff space. We recall that for compact subsets $X$ and $Y$ of a metric space $Z$, the Hausdorff distance between $X$ and $Y$ is

$$
d_{H}(X, Y)=\inf \left\{\epsilon>0 \mid X \subset N_{\epsilon}(Y), Y \subset N_{\epsilon}(X)\right\}
$$

where $N_{\epsilon}(X)=\{z \in Z \mid d(z, X)<\epsilon\}$. If $X$ and $Y$ are compact metric spaces, the Gromov-Hausdorff distance from $X$ to $Y$ is

$$
d_{G H}(X, Y)=\inf _{Z}\left\{d_{H}(X, Y) \mid X, Y \subset Z\right\} .
$$

Let $\mathcal{C M}$ be the set of isometry classes of compact metric spaces with the Gromov-Hausdorff metric. Let $\mathcal{C} \mathcal{M}(n, \rho)$ be the set of all $(X, d) \in \mathcal{C} \mathcal{M}$ 
such that $X$ is a topological $n$-manifold with (topological) metric $d$ with contractibility function $\rho$. We recall that a continuous function $\rho: \mathbb{R}_{+} \rightarrow \mathbb{R}_{+}$with $\rho(0)=0$, continuous at 0 , with $\rho(t) \geq t$ for all $t$ is a contractibility function for a metric space $X$ if there is $R>0$ such that for each $x \in X$ and $t \leq R$, the $t$-ball $B_{t}(x)$ centered at $x$ can be contracted to a point in the $\rho(t)$-ball $B_{\rho(t)}(x)$.

The following theorem is based in part on the results in dimension theory of compacta stated in this paper. It is a combination of results from [52], [74],[33],[35].

6.1. Theorem. (1) Let $X_{k} \rightarrow X$ be a convergent sequence in $\mathcal{C} \mathcal{M}(n, \rho)$. Then there is $N$ such that all $X_{k}$ for $k \geq N$ are homeomorphic to $X_{N}$.

(2) The space $\mathcal{C} \mathcal{M}(n, \rho)$ is not closed in $\mathcal{C M}$. All limit points in $\mathcal{C M} \backslash \mathcal{C M}(n, \rho)$ are infinite dimensional.

(3) Threre are nonhomeomorphic manifolds $M$ and $N$ and a sequence $X_{k} \in \mathcal{C} \mathcal{M}(n, \rho)$ converging to $X \in \mathcal{C} \mathcal{M}$ such that $X_{2 k} \cong M$ and $X_{2 k+1} \cong N$.

The part (3) is based on existance of cell-like maps $f_{M}: M \rightarrow X$ and $f_{N}: N \rightarrow X$ of nonhomeomorphic closed manifolds with the same image.

6.2. Surgery on topological manifolds. The topological surgery structure set on a manifold $M$ is an abelian group which fits into the Sullivan-Wall surgery exact sequence

$$
L_{n+1}\left(\mathbb{Z} \pi_{1}(M)\right) \longrightarrow \mathcal{S}(M) \stackrel{\eta}{\longrightarrow}[M, G / T o p] \stackrel{\theta}{\longrightarrow} L_{n}\left(\mathbb{Z} \pi_{1}(M)\right) .
$$

We note that there are similar surgery structure sets and exact sequences in the PL and Diff category but they are not groups. The set $\mathcal{S}(M)$ of equivalence classes of simple homotopy equivalences $f$ : $N \rightarrow M$ is called the set of topological structures on $M$. Two simple homotopy equivalences of manifolds $f_{1}: N_{1} \rightarrow M$ and $f_{2}: N_{2} \rightarrow M$ are called equivalent if there is a homeomorphism $h: N_{1} \rightarrow N_{2}$ such that $f_{2} \circ h$ is homotopic to $f_{1}$.

In view of the lifting property, every pair of cell-like maps $c_{1}: N \rightarrow$ $X, c_{2}: M \rightarrow X$ induces a homotopy equivalence $f: N \rightarrow M$. The induced homotopy equivalence is unique up to homotopy. If $\operatorname{dim} X<$ $\infty$ and $n \geq 4$, Quinn's uniqueness of resolutions theorem implies that this homotopy equivalence is homotopic to a homeomorphism [83].

For any $X$ one can show that each such homotopy equivalence is simple. By $\mathcal{S}^{C E}(M) \subset \mathcal{S}(M)$ we denote the subset of structures on $M$ realized by cell-like maps as above. One can show that it is a subgroup. 
6.2. Theorem. [33] Let $M^{n}$ be a closed simply connected topological $n$-manifold with finite $\pi_{2}(M), n>5$. Then $\mathcal{S}^{C E}(M)$ is the odd torsion subgroup of $\mathcal{S}(M)$.

Thus a dimension theory phenomenon gives a description of the odd torsion subgroup of the topological surgery structure group in the simply connected case. We refer to [35] for further computations of $\mathcal{S}(M)$ groups.

6.3. Hilbert-Smith conjecture. Here we consider a free action version of the conjecture: A compact group that acts freely on a manifold is a Lie group. The following is an equivalent statement: For all primes $p$ the group $A_{p}$ of p-adic integers cannot act freely on a manifold.

6.3. Theorem (C.T. Yang [95]). Suppose that $A_{p}$ acts freely on a closed $n$-dimensional manifold $M$. Then $\operatorname{dim}_{\mathbb{Z}}\left(M / A_{p}\right)=n+2$.

Proof. We may assume that $M$ is orientable. The group $A_{p}$ is a subgrup of the $p$-adic solenoid $\Sigma_{p}$. We apply Borel's construction to the $A_{p}$ actions on $M$ and $\Sigma_{p}$ to obtain two fibrations

$$
M / A_{p} \stackrel{q_{1}}{\longleftarrow} M \times_{A_{p}} \Sigma_{p} \stackrel{q_{2}}{\longrightarrow} \Sigma_{p} / A_{p}=S^{1} .
$$

Here $q_{2}$ is a locally trivial bundle with the fiber $M$. Since we may assume that the action of $A_{p}$ on $M$ is small, we can regard $q_{2}$ as the trivial bundle. Thus, $M \times_{A_{p}} \Sigma_{p}=M \times S^{1}$. Since $q_{1}$ has the fiber $\Sigma_{p}$ which has trivial cohomology with coefficients in $\mathbb{Z}_{p^{\infty}}, q_{1}$ induces an isomorphism

$$
q_{1}^{*}: H^{n+1}\left(M / A_{p} ; \mathbb{Z}_{p^{\infty}}\right) \rightarrow H^{n+1}\left(M \times S^{1} ; \mathbb{Z}_{p^{\infty}}\right)=\mathbb{Z}_{p^{\infty}} .
$$

Therefore, $\operatorname{dim}_{\mathbb{Z}_{p} \infty} M / A_{p} \geq n+1$. Similarly, one can get that

$$
H^{n+k}\left(M / A_{p}, B ; \mathbb{Z}_{p^{\infty}}\right)=0, \quad k>1
$$

for every closed subset $B \subset M / A_{p}$. Thus, $\operatorname{dim}_{\mathbb{Z}_{p} \infty}\left(M / A_{p}\right)=n+1$.

Since $A_{p}=\lim _{\leftarrow} \mathbb{Z}_{p^{k}}$, the manifold $M$ can be presented as the inverse limit of the orbit spaces $M / p^{k} A_{p}$ with $p-t o-1$ coverings as the bonding maps. Since multiplication by $p$ is an isomorphism for $\mathbb{Q}$ and $\mathbb{Z}_{q}$ for prime $q \neq p$ every bonding map induces a monomorphism of cohomology with coefficients in $\mathbb{Z}_{q}, q \neq p$, and in $\mathbb{Q}$. This implies that $\operatorname{dim}_{\mathbb{Z}_{q}}\left(M / A_{p}\right)=n$ and $\operatorname{dim}_{\mathbb{Q}}\left(M / A_{p}\right)=n$. This information completely defines the dimension type $D_{M / A_{p}}$. Then

$$
\operatorname{dim}_{\mathbb{Z}}\left(M / A_{p}\right)=\max _{r \in \mathcal{P}}\left\|D_{M / A_{p}}(r)\right\|=\operatorname{dim}_{\mathbb{Z}_{p} \infty}\left(M / A_{p}\right)+1=n+2 .
$$


6.4. Boundaries of groups. The Bestvina-Mess formula connects the virtual cohomological dimension of hyperbolic groups with the cohomological dimension of its Gromov's boundary.

6.4. Theorem. [9] For a hyperbolic group $\Gamma$,

$$
\operatorname{vcd}_{F} \Gamma=\operatorname{dim}_{F} \partial \Gamma+1 .
$$

Here $v c d_{F}$ stands for the virtual cohomological dimension with the coefficient group $F[12]$. Bestvina extended this formula for groups with $Z$-boundary [8]. His formula allows to construct examples of Coxeter groups $\Gamma_{1}$ and $\Gamma_{2}$ with

$$
\operatorname{vcd}\left(\Gamma_{1} \times \Gamma_{2}\right)<\operatorname{vcd}\left(\Gamma_{1}\right)+\operatorname{vcd}\left(\Gamma_{2}\right)
$$

for integers as the coefficients [28]. In view of Bestvina's formula the construction of such groups is reduced to a construction of groups with boundaries which are not dimensionally full-valued.

Boundaries of hyperbolic groups are so called Markov's compacta [79], i.e. compacta constructed as inverse limits of simplicial complexes with bonding maps that consist of finitely many building blocks (see [32][79] for the definition). It is an open problem what kind of dimension function can be realized by Markov's compacta.

The following theorem implies that the dimension type realized by boundary of a Coxeter group is dimensionally regular.

\subsection{Theorem. [28] For every Coxeter group}

$$
\operatorname{vcd}(\Gamma \times \Gamma)=2 v c d(\Gamma) .
$$

6.5. Large scale dimension. M. Gromov introduced the notion of asymptotic dimension to study large scale geometry of discrete groups [55]. This concept sparked a great attention when Guoliang Yu proved the Higher Signature Novikov Conjecture for manifolds whose fundamental group has finite asymptotic dimension [96].

6.6. Definition. The asymptotic dimension of a metric space $X$ does not exceed $n$, asdim $X \leq n$ if for every uniformly bounded cover $\mathcal{U}$ of $X$ there is a larger uniformly bounded cover $\mathcal{V}, \mathcal{U} \prec \mathcal{V}$, of multiplicity $\leq n+1$. We say that $\operatorname{asdim} X=n$ if $\operatorname{asdim} X \leq n$ and the condition $\operatorname{asdim} X \leq n-1$ does not hold true.

Since this definition is symmetric to that for the covering dimension, Theorem 2.2 has an asymptotic analog:

6.7. Theorem. For a metric space $X$ the following are equivalent - (1) $\operatorname{asdim} X \leq n$; 
- (2) Given $R<\infty$, there is a uniformly bounded cover $\mathcal{U}=$ $\mathcal{U}^{0} \cup \cdots \cup \mathcal{U}^{n}$ of $X$ such that each family $\mathcal{U}^{i}$ consists of $R$-disjoint open sets;

- (3) Given $\epsilon<0, X$ admits an $\epsilon$-Lipschitz uniformly co-bounded map to an $n$-dimensional subcomplex of the unit simplex $\Delta \subset \ell_{2}$ in the Hilbert space;

- (4) $X$ is is the image of a metric space $Y$ with $\operatorname{asdim} Y=0$ under a map $f: Y \rightarrow X$ that satisfies the condition $(B)_{n+1}$ : For each $r<\infty$, there exists $d<\infty$ so that for each subset $B \subset Y$ with $\operatorname{diam}(B)<r, f^{-1}(B)=\cup_{i=1}^{n} A_{i}$ for some subsets $A_{i} \subset X$ with $\operatorname{diam}\left(A_{i}\right)<d$ for $i=1, \ldots, n$;

- (5) X admits an anti-Čech approximation by $n$-dimensional polyhedra.

The equivalence of these conditions can be derived from [55], [5], [6], [73]. Here (4) and (5) are analogous to (8) and (9) from Theorem 2.2. The notion of an anti-Čech approximation was introduced by Higson and Roe by analogy with the Cech approximation of compacta by polyhedra [58]. Asymptotic analogs of conditions (5)-(7) of Theorem 2.2 are missing. An analog of (4) can be proven under assumption of finite dimensionality.

The following theorem connects asdim and dim.

6.8. Theorem. For a proper metric space $X$,

(1) [36] $\operatorname{asdim} X \leq \operatorname{dim} \nu X$ where $\nu X$ is the Higson corona of $X$.

(2) [30] $\operatorname{asdim} X=\operatorname{dim} \nu X$ provided $\operatorname{asdim} X<\infty$.

We recall that the Higson corona $\nu X$ of a proper metric space $X$ is the remainder of the compactification of $X$ defined by the ring of bounded functions $f: X \rightarrow \mathbb{R}$ satisfying the condition: For any $r<\infty$,

$$
\lim _{x \rightarrow \infty} \operatorname{diam}\left(f\left(B_{r}(x)\right)\right)=0
$$

where $B_{r}(x)$ denotes the $r$-ball centered at $x$.

The asymptotic dimension theory of asymptotically infinite dimensional metric spaces is of special interest. The reason for such interest is that even for asymptotically infinite dimensional groups some tameness conditions like asymptotic property $\mathrm{C}$ lead to a proof of Novikov type conjectures for the groups satisfying those conditions [30]. One of such relatively recent conditions is called the finite decomposition complexity [56],[84],[44]. It is still unclear what is its analog for compacta.

\section{REFERENCES}

[1] Alexandroff, P. S. Analyse géométrique de la dimension des ensambles fermés, C. R. Acad. Paris 191 (1930), 475-477. 
[2] Alexandroff, P. S. Dimensionstheorie, Math. Ann. 106 (1932), 161-238.

[3] Amarasinghe, A.; Dranishnikov, A. On homology of complements of compact sets in Hilbert cube, Topology Appl. 221 (2017), 262-269.

[4] Ancel, F. D. Proper hereditary shape equivalences preserve property C. Topology Appl. 19 (1985), no. 1, 71-74.

[5] Bell, G. C.; Dranishnikov, A. N. A Hurewicz-type theorem for asymptotic dimension and applications to geometric group theory. Trans. Amer. Math. Soc. 358 (2006), no. 11, 4749-4764.

[6] Bell G., Dranishnikov, A. Asymptotic dimension. Topology Appl. 155 (2008), no. $12,1265-1296$.

[7] Bestvina, M. Characterizing k-dimensional universal Menger compacta. Mem. Amer. Math. Soc. 71 (1988), no. 380.

[8] Bestvina, M. Local homology properties of boundaries of groups. Michigan Math. J. 43 (1996), no. 1, 123-139.

[9] Bestvina, M.; Mess, G. The boundary of negatively curved groups. J. Amer. Math. Soc. 4 (1991), no. 3, 469-481.

[10] Boltyanskii, V. An example of a two-dimensional compactum whose topological square is three-dimensional. (Russian) Doklady Akad. Nauk SSSR (N.S.) 67, (1949). 597-599 (English translation in Amer. Math. Soc. Translation 1951, (1951). no. 48, 3-6).

[11] Bowers, P. L. General position properties satisfied by finite products of dendrites. Trans. Amer. Math. Soc. 288 (1985), no. 2, 739-753.

[12] K. Brown, Cohomology of groups, Springer 1982.

[13] Brouwer, L .E. J. Beweis der Invariantz der Dimensionenzahl, Math. Ann. 70 (1911), 161-165.

[14] Brouwer, L .E. J. Über den natürlichen Dimensionsbegriff, J. reine angew. Math. 142 (1913), 146-152.

[15] Brouwer, L .E. J. Über den natürlichen Dimensionsbegriff, Proc. Akad. Amsterdam 26 (1923), 795-800.

[16] Brouwer, L .E. J. Berichtigung zur Abhandlung "Über den natürlichen Dimensionsbegrff", dieses Journal Bd. 142 (1913), SW. 146-152, Journ. für die reine und angew. Math. 151 (1924), 253.1

[17] Brouwer, L .E. J. Zum natürlichen Dimensionsbegriff, Math. Zeitschr. 21 (1924), 312-314.

[18] Brouwer, L .E. J. Bemerkungen zum natürlichen Dimensionsbegriff, Proc. Akad. Amsterdam 27 (1924), 635-638.

[19] Dranishnikov, A. N. Universal Menger compacta and universal mappings. (Russian) Mat. Sb. (N.S.) 129(171) (1986), no. 1, 121-139.

[20] Dranishnikov, A. N. On homological dimension modulo $p$. (Russian) Mat. Sb. (N.S.) 132(174) (1987), no. 3, 420-433, 446; translation in Math. USSR-Sb. 60 (1988), no. 2, 413-425

[21] Dranishnikov, A. N. On a problem of P. S. Aleksandrov. (Russian) Mat. Sb. (N.S.) 135(177) (1988), no. 4, 551-557, 560; translation in Math. USSR-Sb. 63 (1989), no. 2, 539-545.

[22] Dranishnikov, A. N. Homological dimension theory. Russian Math. Surveys 43 (4) (1988), 11-63.

[23] Dranishnikov, A. N. Generalized cohomological dimension of compact metric spaces. Tsukuba J. Math. 14 (1990), no. 2, 247-262. 
[24] Dranishnikov, A. N. Stable cohomotopy dimension and weakly infinitedimensional spaces. Topology Appl. 47 (1992), no. 1, 79-81.

[25] Dranishnikov, A. N. The power substitution for rings of complex and real functions on compact metric spaces. Proc. Amer. Math. Soc. 123 (1995), no. 9, 2887-2893.

[26] Dranishnikov A. N. On the mapping intersection problem. Pacific J. Math. 173 (1996), 403-412.

[27] Dranishnikov, A. N. On Chogoshvili's conjecture. Proc. Amer. Math. Soc. 125 (1997), no. 7, 2155-2160.

[28] Dranishnikov, A. N. On the virtual cohomological dimensions of Coxeter groups. Proc. Amer. Math. Soc. 125 (1997), no. 7, 18851891.

[29] Dranishnikov, A. N. On the dimension of the product of two compacta and the dimension of their intersection in general position in Euclidean space. Trans. Amer. Math. Soc. 352 (2000), no. 12, 5599-5618.

[30] Dranishnikov, A. N. Asymptotic topology. (Russian) Uspekhi Mat. Nauk 55 (2000), no. 6(336), 71-116; translation in Russian Math. Surveys 55 (2000), no. $6,1085-1129$.

[31] Dranishnikov, A N. Cohomological dimension theory of compact metric spaces, Topology Atlas invited contribution, Vol 6, 2001, http://at.yorku.ca/topology.taic.html (see also arXiv:math/0501523).

[32] Dranishnikov, A. N. Cohomological dimension of Markov compacta. Topology Appl. 154 (2007), no. 7, 1341-1358.

[33] Dranishnikov, A.; Ferry, S. Cell-like maps and topological structures, Preprint MPI 2006-114, www.mpim-bonn.mpg.de/preprints.

[34] Dranishnikov, A. N.; Ferry, S. C.; Weinberger, S. Large Riemannian manifolds which are flexible. Ann. of Math. (2) 157 (2003), no. 3, 919-938.

[35] Dranishnikov, A. N.; Ferry, S. C.; Weinberger, S. An infinite-dimensional phenomenon in finite dimensional metric topology, (2016), preprint.

[36] Dranishnikov, A. N.; Keesling, J.; Uspenskij, V. V. On the Higson corona of uniformly contractible spaces. Topology 37 (1998), no. 4, 791-803.

[37] Dranishnikov, A.; Levin, M. Dimension of the product and classical formulae of dimension theory. Trans. Amer. Math. Soc. 366 (2014), no. 5, 2683-2697.

[38] Dranishnikov, A.; Levin, M. On dimensionally exotic maps. Israel J. Math. 201 (2014), no. 2, 967-987.

[39] Draninikov, A. N.; Repovs, D. On unstable intersections of 2-dimensional compacta in Euclidean 4-space. Topology Appl. 54 (1993), no. 1-3, 3-11.

[40] Draninikov, A. N.; Repovs, D.; Shchepin, E. V. On intersections of compacta of complementary dimensions in Euclidean space. Topology Appl. 38 (1991), no. 3, 237-253.

[41] Dranishnikov, A. N.; Repovs, D.; Shchepin, E. V. Dimension of products with continua. Topology Proc. 18 (1993), 57-73.

[42] Dranishnikov, A. N.; West, J. On compacta that intersect unstably in Euclidean space. Topology Appl. 43 (1992), no. 2, 181-187.

[43] Dranishnikov, A. N.; West, J. E. Correction to: "Compact group actions that raise dimension to infinity" [Topology Appl. 80 (1997), no. 1-2, 101114; MR1469471]. Topology Appl. 135 (2004), no. 1-3, 249252.

[44] Dranishnikov, A.; Zarichnyi, M. Asymptotic dimension, decomposition complexity, and Haver's property C. Topology Appl. 169 (2014), 99-107. 
[45] Dobrowolski, T.; Levin, M.; Rubin, L. R. Certain 2-stable embeddings. Topology Appl. 80 (1997), no. 1-2, 81-90.

[46] Dydak, Jerzy Cohomological dimension and metrizable spaces. Trans. Amer. Math. Soc. 337 (1993), no. 1, 219-234.

[47] Dydak, Jerzy Cohomological dimension and metrizable spaces. II. Trans. Amer. Math. Soc. 348 (1996), no. 4, 1647-1661.

[48] Dydak, J.; Walsh. J. Infinite-dimensional compacta having cohomological dimension two: an application of the Sullivan conjecture. Topology 32 (1993), no. 1, 93-104.

[49] Engelking, Ryszard Theory of dimensions finite and infinite. Sigma Series in Pure Mathematics, 10. Heldermann Verlag, Lemgo, 1995.

[50] Fedorchuk, V. V.; Levin, M.; Shchepin, E. V. On the Brouwer definition of dimension. Russian Math. Surveys 54 (1999), no. 2, 432-433.

[51] Fedorchuk, V. V.; Ivanov, A. V., van Mill, J. Intermediate dimensions of products, Topology Appl. 153 (2006) 3265-3276.

[52] Ferry, S. Homotoping $\epsilon$-maps to homeomorphisms. Amer. J. Math. 101 (1979), no. $3,567-582$.

[53] Gabai D., On the topology of ending lamination space. Geom. Topol. 18 (2014), no. 5, 2683-2745.

[54] Garity, D.; Wright, D. Vertical order in the Hilbert cube, Illinois Journ. of Math. 31 No 3 (1987), 446-452.

[55] M. Gromov, Asymptotic invariants of infinite groups, Cambridge University Press, Geometric Group Theory, vol 21993.

[56] Guentner, Erik; Tessera, Romain; Yu, Guoliang A notion of geometric complexity and its application to topological rigidity. Invent. Math. 189 (2012), no. 2, 315-357.

[57] Henderson, D. W. Each strongly infinite dimensional compactum contains a hereditary infinite dimensional compact subset, Amer. Journ. of Math. 89 (1967), 122-123.

[58] Higson, Nigel; Roe, John Analytic K-homology. Oxford Mathematical Monographs. Oxford Science Publications. Oxford University Press, Oxford, 2000.

[59] Hurewicz W. Über dimensionserhöhende stetige Abbildungen, J. reine angew. Math. 1969 (1932), 71-78.

[60] Kodama, Y. Test spaces for homological dimension. Duke Math. J. 29 (1962) $41-50$.

[61] Kolmogorov, A. N. Über offene Abbildungen, Ann. of Math. (2) 38 (1937), 36-38.

[62] Koyama, A.; Krasinkiewicz, J.; Spie, S. Embeddings into products and symmetric productsan algebraic approach. Houston J. Math. 38 (2012), no. 2, 611-641.

[63] Kroonenberg, N. Characterization of finite-dimensional Z-sets. Proc. Amer. Math. Soc. 43 (1974), 421-427.

[64] Kuzminov, V. I. Homological dimension theory. Russian Math Surveys 23 (5) (1968), 1-45.

[65] Levin, M. Resolving rational cohomological dimension via a Cantor group action. Algebr. Geom. Topol. 15 (2015), no. 4, 2427-2437.

[66] Levin, M. On compacta not admitting a stable intersection in $R^{n}$, Preprint, 2013, arXiv:1310.2091. 
[67] Levin, M. A dimensional property of Cartesian product. Fund. Math. 220 (2013), no. 3, 281-286.

[68] Levin, M. Rational acyclic resolutions. Algebr. Geom. Topol. 5 (2005), 219-235.

[69] Levin, M. Universal acyclic resolutions for arbitrary coefficient groups. Fund. Math. 178 (2003), no. 2, 159-169.

[70] Levin, M. A Z-set unknotting theorem for Nbeling spaces. Fund. Math. 202 (2009), no. 1, 1-41.

[71] Levin, M.; Shapira, U.; Weiss, B. Closed orbits for the diagonal group and well-rounded lattices, Preprint 2014, arXiv:1405.5682.

[72] Mardešic̀, S. Compact subsets of $\mathbb{R}^{n}$ and dimension of their projections, Proc. AMS. 41 (1973), 631-633.

[73] Miyata, Takahisa; Virk, Žiga Dimension-raising maps in a large scale. Fund. Math. 223 (2013), no. 1, 8397.

[74] Moore, T. E. Gromov-Hausdorff convergence to nonmanifolds. J. Geom. Anal. 5 (1995), no. 3, 411-418.

[75] Nöbeling, G. Die Projektionen einer kompakten, n-dimensionalen Menge im $R_{k}$, Ergebnisse eines math. Koll. 4 (1932), 24-25.

[76] van Mill, Jan; Pol, Roman, An example concerning the Menger-Urysohn formula. Proc. Amer. Math. Soc. 138 (2010), no. 10, 3749-3752.

[77] Nagórko, A. Characterization and topological rigidity of Nbeling manifolds. Mem. Amer. Math. Soc. 223 (2013), no. 1048

[78] Ostrand, Phillip A. Dimension of metric spaces and Hilbert's problem 13. Bull. Amer. Math. Soc. 71 (1965), 619-622.

[79] Pawlik, D. Gromov boundaries as Markov compacta 2015, Preprint, arXiv:1503.04577.

[80] Poincaré, H. Pourquoi l'espace a trois dimensions, Revue de Metaphysique et de Morale, 20 (1912), 484.

[81] Pol, R. A weakly infinite-dimensional compactum which is not countabledimensional. Proc. Amer. Math. Soc. 82 (1981), no. 4, 634-636.

[82] Pontryagin L. S. Sur une hypothese fondamentale de la theorie de la dimension. C. R. Acad. Sci. Paris 190 (1930), 1105-1107.

[83] F. Quinn, Ends of maps. I, Ann. of Math.(2), 110 No 2, (1979), 275-331.

[84] Ramras, Daniel A.; Tessera, Romain; Yu, Guoliang Finite decomposition complexity and the integral Novikov conjecture for higher algebraic K-theory. J. Reine Angew. Math. 694 (2014), 129-178.

[85] Raymond, F; Williams, R. F. Examples of p-adic transformation groups. Bull. Amer. Math. Soc. 66 (1960), 392-394.

[86] Raymond, F. and Williams, R. F. Examples of p-adic transformation groups, Ann. of Math. (2) 78 (1963), 92-106.

[87] Shchepin, E. V. Arithmetic of dimension theory. Russian Math. Surveys 53 (1998), no. 5, 975-1069.

[88] Spież, S. Imbeddings in $\mathbb{R}^{2 m}$ of $m$-dimensional compacta with $\operatorname{dim}(X X)<2 m$. Fund. Math. 134 (1990), no. 2, 105-115.

[89] Sternfeld, Y. Hilbert's 13th problem and dimension. Geometric aspects of functional analysis (1987-88), 1-49, Lecture Notes in Math., 1376, Springer, Berlin, 1989.

[90] Sternfeld, Y. Linear superpositions with mappings which lower dimension. Trans. Amer. Math. Soc. 277 (1983), no. 2, 529-543. 
[91] R.M. Switzer, Algebraic Topology - Homotopy and Homology, Springer, 1975.

[92] Walsh, J. Dimension, cohomological dimension, and cell-like mappings, Lecture Notes Math. 870 (1981), 105-118.

[93] Williams, R. F. A useful functor and three famous examples in topology. Trans. Amer. Math. Soc. 106 (1963), 319-329.

[94] Williams, R. F. The construction of certain 0-dimensional groups, Trans. Amer. Math. Soc. 129 (1967), 140-156.

[95] Yang C.T. p-Adic transformation groups, Mich math. J. 7 (1960), 201-218.

[96] Yu, G. The Novikov conjecture for groups with finite asymptotic dimension. Ann. of Math. (2) 147 (1998), no. 2, 325-355.

[97] Zapletal, J. Dimension theory and forcing, Topology and Its Applications 167 (2014), 31-35.

Alexander N. Dranishnikov, Department of Mathematics, UniverSity of Florida, 358 Little Hall, Gainesville, FL 32611-8105, USA

E-mail address: dranish@math.ufl.edu 